\title{
Editorial
}

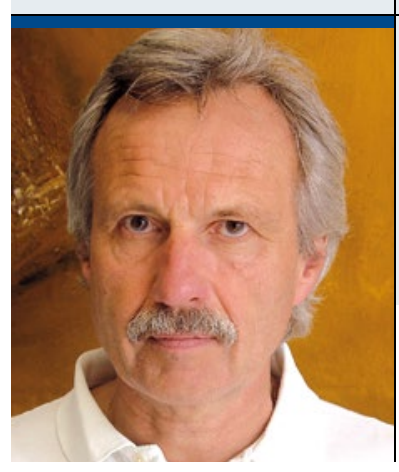

„Viagra für Frauen. Wäre es nicht billiger, sich den Partner schön

zu trinken? Die Nebenwirkungen sind die gleichen."

\section{Quergelesen zum Jahresschluss}

V iagra für Frauen! Auf Druck der Lobbyisten wurde es von der US-amerikanischen Food and Drug Administration (FDA) zugelassen. Flibanserin heißt der Wirkstoff. Als Antidepressivum auf den Markt gebracht, erwies es sich als wenig wirksam. Bei den weiblichen Teilnehmern einer klinischen Studie stimulierte Flibanserin nicht die Lustigkeit, aber die Lust auf den Partner. Wenngleich nicht ohne Nebenwirkungen: Übelkeit, Schwindel und Kopfschmerzen können auftreten. Wäre es nicht billiger, sich den Partner schön zu trinken? Die Nebenwirkungen sind die gleichen.

Early to bed and early to rise makes a man happy, healthy and wise. Und obendrein schlank, glaubt man den Ergebnissen einer Langzeitstudie der University of California. Die Forscher wollen einen Zusammenhang zwischen spätem Nachtschlaf und einem hohen Body-Mass-Index (BMI) festgestellt haben. Eigentlich kein Wunder. Wer spät zu Bett geht, hat länger Zeit zum Chips und Burger futtern. „Gemeinsam klug entscheiden“ heißt die neue Losung der Arbeitsgemeinschaft der Wissenschaftlichen Medizinischen Fachgesellschaften (AWMF). Gemeint ist eine partizipative Entscheidungsfindung des Arztes und des informierten Patienten. Gemeinsam sollen sie „auf Augenhöhe“ über eine anstehende Diagnostik und Therapie entscheiden. Und nicht nur das. Das Ergebnis soll sogar gemeinsam verantwortet werden. Hört sich an wie „Schweine im Weltall“. Bis der liebe Doktor für diese Entscheidungsfindung auf „Augenhöhe“ seinen Patienten geschult hat, wird wohl ein "Crashkurs“ von einer Woche kaum genügen. Sicher kein Mittel gegen Ärztemangel. Und nach einem etwaigen Eingriff wird die erste Frage des Patienten sein: Sagen Sie mal, Herr Doktor, was haben WIR eigentlich operiert? Ganz abgesehen von der gemeinsamen Verantwortung bei Ergebnisversagern. Die anstehende Gerichtsposse ist „Oskar“-verdächtig.

„Reha rechnet sich“, jubiliert die Deutsche Rentenversicherung (DRV) und sieht ihre Arbeitsplätze gesichert. Die Rehabilitation erhalte die Fähigkeit und
Fertigkeit zur Selbstversorgung der Patienten. Das mag nach Operationen zutreffen. Bei den sogenannten Heilverfahren für chronisch-degenerative Erkrankungen, zumeist ein Ganzkörper-Kreuz-Gliederschmerz, verwundert das. Denn das DRV-Motto "Reha vor Rente" hört sich probandenmodifiziert anders an: „Rente nach Reha“. Und wehe, wenn nicht! Dann hagelt es Beschwerden über die Reha-Einrichtung und ihre Ärzte, die von der Rentenversicherung - nonchalant und unbürokratisch - mit der Anforderung einer chefärztlichen Stellungnahme an die Reha-Einrichtung durchgereicht werden. $\mathrm{Zu}$ Zeiten des Massage-Fango-Tango-Booms inklusive Kurschatten war der Proband gut gelaunt und topfit. Allenfalls traurig, dass es vorüber und etwas schuldbewusst, dass es gewesen.

Die Juristen bestimmen die Medizin. In bekannter Tendenz der Krankenkassenliebkosung urteilten die Robenträger des 1. Senats des Bundessozialgerichts, dass die prästationäre Behandlung nicht abrechenbar ist, wenn die vertragsärztliche Diagnostik nicht ausgeschöpft ist. Bis zum Beispiel eine präoperative Diagnostik zwischen den operativen und konservativen Fachrichtungen abgestimmt und erfüllt ist, hat sich der Patient gegebenenfalls seiner Spielballfunktion zwischen den Instanzen durch Ableben entzogen. Aus Kassensicht erfreulich, aus Juristensicht schicksalhaft.

„Humor ist der Knopf, der verhindert, dass einem der Kragen platzt“ (Joachim Ringelnatz). Die Redaktion wünscht Ihnen frohe Festtage - und für 2016 Gesundheit sowie den notwendigen Humor!

Ihr

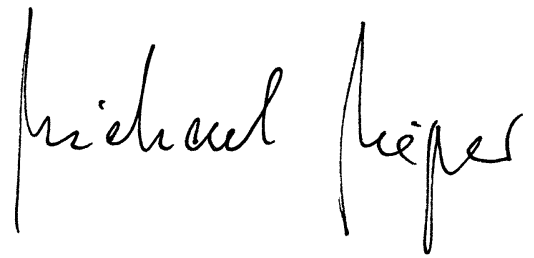

